\title{
A Wheel-based Stair-climbing Robot with a Hopping Mechanism
}

\author{
Koki Kikuchi, Naoki Bushida, Keisuke Sakaguchi, Yasuhiro Chiba, \\ Hiroshi Otsuka, Yusuke Saito, Masamitsu Hirano and Shunya Kobayashi \\ Chiba Institute of Technology \\ Japan
}

\section{Introduction}

In this chapter, we introduce a stair-climbing robot developed in our laboratory. This robot consists basically of two body parts connected by springs, and hops as a result of the vibration of a two-degrees-of-freedom (2-DOF) system. The excellent combination between the frequencies of the robotic body vibration and the tread-riser interval of stairs enables a small and simple robot fast stair climbing, soft landing, and energy saving.

In an attempt to give the robot mobility in an environment such as an office building having steps and stairs, various mechanisms have been proposed and developed. Each one of which has different characteristics. For example, wheel-based robots are very simple in terms of both mechanical design and control, and they can travel quickly and stably. But their size tends to be big for climbing stairs, as they cannot surmount a riser higher than their wheel radius. On the other hand, although crawler-type robots can climb over a riser higher than a wheel-based robot, they are slow and noisy. Typical examples of crawler-type robots are TAQT (Hirose et al., 1992) that can carry a human and Kenaf (Yoshida et al., 2007) for rescue operations. Legged robots, especially humanoid ones, are well suited for climbing stairs, but require many DOFs and complex control. Honda's ASIMO (ASIMO OFFICIAL SITE), AIST's HRP (Harada et al., 2006) and Waseda University's legged robot (Sugahara et al., 2007) are good examples. In addition, the hybrids of these types have been proposed and have improved upon mutual demerits. Chari-be (Nakajima et al., 2004), with two wheels and four legs, travels quickly on its wheels over flat terrain, and climbs using its legs in rough terrain such as a step and stairs. A biped-type robot with a wheel at the tip of its legs (Matsumoto et al., 1999) climbs stairs smoothly. RHex (Altendorfer et al., 2001) has six compliant rotary legs and travels speedily not only up and down stairs, but also even uncertain terrain such as a swamp. Moreover, modular robots such as an articulated snakelike robot and special mechanisms for stairs have also been proposed. Yim's snake-like robot (Yim et al., 2001) climbs stairs, transforming its own loop form into a stair shape. These excellent mechanisms have improved the manoeuvrability of the robot for rough terrain, but as most are general-purpose robots for rough terrain, a more specialized mechanism must be developed if we focus solely on stair-climbing ability in an office building. 
From this point of view, we have developed a stair-climbing robot specifically for use in an office building (Sakaguchi et al., 2007, Asai et al. 2008 \& Kikuchi et al., 2008). Our robot cannot climb stairs with various-height or irregular risers smoothly, but it does climb stairs with a priori determined regular risers rapidly (less than $1.0 \mathrm{~s}$ per step), softly (less than the impact at takeoff, at the landing point), and economically. Furthermore, the mechanical design and control are quite simple, and additionally the height of the robot is almost the same as the common stair riser. These features are very important for practical tasks such as monitoring the situation in an office. Here, we introduce the hopping mechanism and property, and show the experimental result of the fast stair climbing and soft-landing.

\section{Hopping mechanism and robotic design}

The mathematical hopping model consists of two mass points, $m_{1}$ and $m_{2}$, connected by a spring, as shown in Fig. 1. The lower mass point, $m_{2}$, hops if, and only if, the lifting force provided by the spring, $k\left(z_{1}-z_{2}\right)$, and the wire, $T_{\mathrm{w}}$, exceeds the force of gravity on the lower mass, $m_{2} g$. The trajectories of the two mass points during hopping evolve based on the reduced mass, the mass ratio between the upper and lower masses, spring constant, $k$, the friction of the shaft, and stored spring energy. Figure 2 shows a manufactured robot with this hopping mechanism. The robot consists of an upper body part (Body 1) and a lower body part (Body 2) connected by four springs and a wire. Here, the upper body part has a CPU (H8/tiny) for control, a receiver, a position sensitive detector (PSD), a reel with a gear and a motor, a solenoid, and batteries (CPU: $7.4 \mathrm{~V}$, motor: $30 \mathrm{~V}$ for the reel and $22.2 \mathrm{~V}$ for the travel, and solenoid: $44.4 \mathrm{~V})$. The lower body part has two motors for translational travel, four 56-mm diameter wheels, four shafts and two acceleration sensors for the $\mathrm{z}$ direction. The robot first stores the spring energy by reeling in the wire. The reel mechanism is then detached by the solenoid, and the robot hops by releasing the stored spring energy. Here, body parts 1 and 2 correspond to the mass points of the two-dimensional mathematical model shown in Fig. 1. The robot is $370 \mathrm{~mm}$ tall, $155 \mathrm{~mm}$ wide and $140 \mathrm{~mm}$ long. Also, the robot has no suspension to act as a damper, as we scrutinize the impact acceleration of body parts.

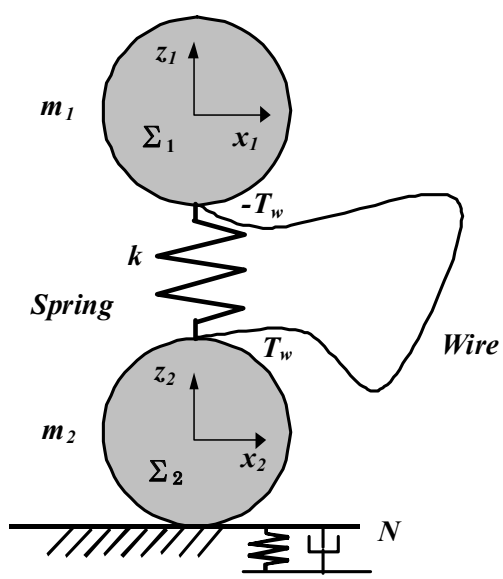

Fig. 1. Two-dimensional mathematical model of hopping mechanism 


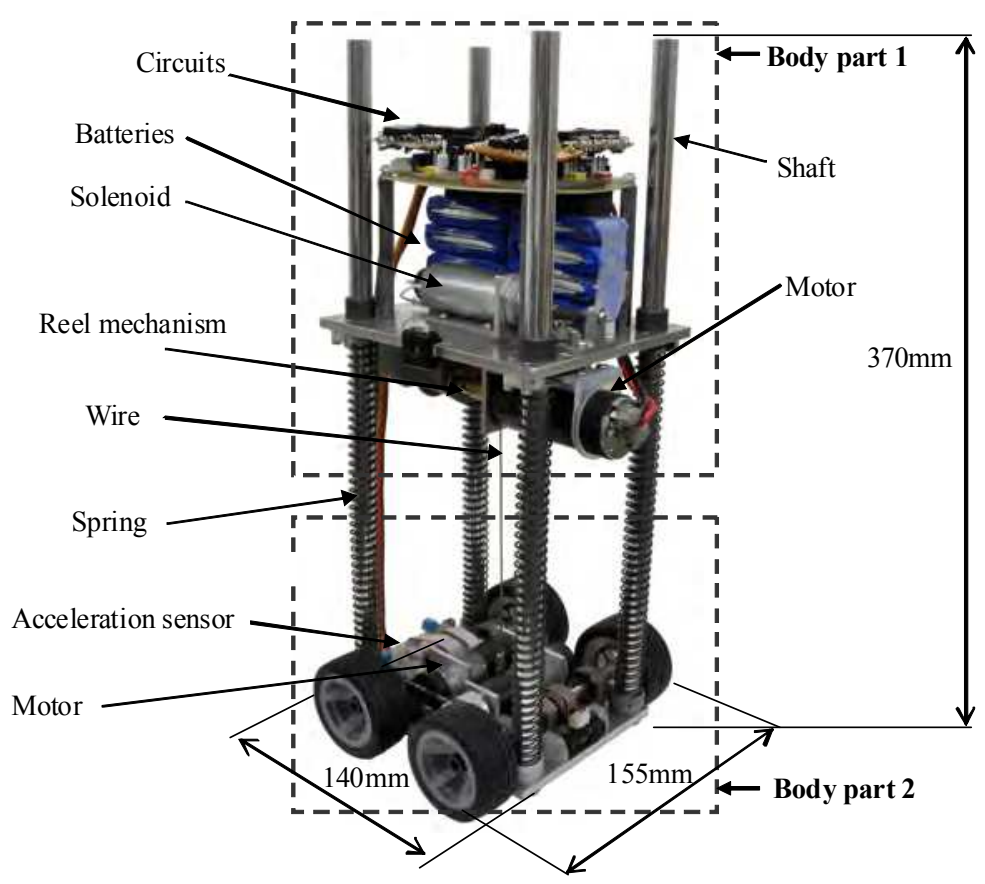

Fig. 2. Wheel-based robot with hopping mechanism

\section{Mathematical model for simulation}

The robot is modeled simply as a 2-DOF spring-mass system. If the posture of the robot can be neglected, the equations of motion are given by

$$
\begin{array}{r}
\left(m_{1}+m_{2}\right) x_{1}{ }^{\prime \prime}=f_{\mathrm{x}} \\
m_{1} \mathrm{z}_{1}{ }^{\prime \prime}+k\left(z_{1}-z_{2}\right)=-m_{1} \mathrm{~g}-T_{\mathrm{w}}-\mu_{\mathrm{t}} F_{\mathrm{f}} \\
m_{2} \mathrm{z}_{2}{ }^{\prime}+k\left(z_{2}-z_{1}\right)=-m_{2} \mathrm{~g}+T_{\mathrm{w}}+\mu_{\mathrm{t}} F_{\mathrm{f}}+N
\end{array}
$$

where $\left(x_{1}, z_{1}\right)$ and $\left(x_{2}, z_{2}\right)$ are coordinates for each body part $\left(z_{1}=z_{2}\right.$ at the natural length of the spring), $m_{1}$ and $m_{2}$ are the masses of body parts 1 and $2, k$ is the spring constant, $f_{\mathrm{x}}$ is the motor force for horizontal travel, $\mu_{\mathrm{t}} F_{\mathrm{f}}$ is the friction of the shaft (the magnitude of $F_{\mathrm{f}}$ is determined by the pilot experiment and the sign is determined by the relative vertical velocity between the two masses, $\mathrm{d}\left(z_{2}-z_{1}\right) / \mathrm{dt}$ ), and $N$ is the reaction of the ground. The ground is simply modeled by a spring and a damper, as shown Fig. $1 . T_{\mathrm{w}}$, the wire tension is a positive value or zero for tensional or relaxant conditions, respectively. Note that as the posture of the robot is neglected for simplification, $x_{1}$ is always equal to $x_{2}$.

Here, the condition for takeoff of lower mass, $z_{2}$, is given by

$$
m_{2} \mathrm{~g}<k\left(z_{1}-z_{2}\right)+T_{\mathrm{w}}-\mu_{\mathrm{t}} F_{\mathrm{f}}
$$

Assuming that the friction of shaft, $\mu_{\mathrm{t}} F_{\mathrm{f}}$, is neglected $\left(F_{\mathrm{f}}=0\right)$ and that we do not control the wire tension $\left(T_{\mathrm{w}}=0\right)$ during hopping, the trajectories of the two masses at the time $t$ after takeoff, i.e., during hopping $\left(f_{\mathrm{x}}=0, N=0\right.$, and $\left.z_{2}>0\right)$, are as follows, 


$$
\begin{array}{r}
x_{1}=x_{2}=v_{x} t+D \\
z_{1}=h M / m_{1} \sin (\omega t+\varphi)-g(t-T)^{2} / 2+C \\
z_{2}=-h M / m_{2} \sin (\omega t+\varphi)-g(t-T)^{2} / 2+C \\
\omega=(k / M)^{0.5}, M=1 /\left(1 / m_{1}+1 / m_{2}\right)
\end{array}
$$

where $v_{\mathrm{x}}$ is the horizontal velocity at takeoff, $D$ is the starting point of takeoff, $h, \varphi, T$, and $C$ are constants determined by the initial conditions, $\omega$ is the angular frequency, and $M$ is the reduced mass between the two masses. Hence, the horizontal velocity is constant during hopping. The air resistance is neglected. The first terms on the right side of the $\mathrm{z}$ equations, Eq. (3b), represent vibration caused by the two body parts. Although two natural frequencies must exist because the system has a 2-DOF configuration, the lower natural frequency is zero in this case. The second terms on the right side of the $\mathrm{z}$ equations represent the parabolic motion of the center of mass (COM). Consequently, the hopping motion of the robot after takeoff is represented by the combination of the vibration of the mass points and the parabolic motion of the COM. Hence, the point at which the velocity in the $\mathrm{z}$ direction of the vibration of body part 2 and that of the parabolic motion of the COM are canceled out, is the soft-landing point. For later discussion, we define "soft-landing" as a landing in which the vertical velocity of the lower body part is zero $\left(\mathrm{z}_{2}^{\prime} \approx 0\right)$, the acceleration is zero $\left(\mathrm{z}_{2}{ }^{\prime \prime} \approx 0\right)$, and the third differential is zero or negative $\left(\mathrm{z}_{2}^{\prime \prime \prime} \leq 0\right)$ at landing height, $H$ (where $H$ is the riser height). Figure 3 shows two typical examples of the trajectories of the mass points, $z_{1}$ and $z_{2}$, during hopping. The red dashed line depicts the hopping for the higher riser, and the blue solid line is that for the lower riser. The points " $\mathrm{A}$ " and " $\mathrm{B}$ " are ideal landing points, i.e., mathematical stationary and inflection points. These robots can softly climb stairs with risers of $H=0.1[\mathrm{~m}]$ and $H=0.2[\mathrm{~m}]$, respectively. Note that the closer the $\mathrm{z}_{2}{ }^{\prime \prime \prime}$ is to zero, the lower the landing impact for noise, because the lower body part can fly parallel to the tread.

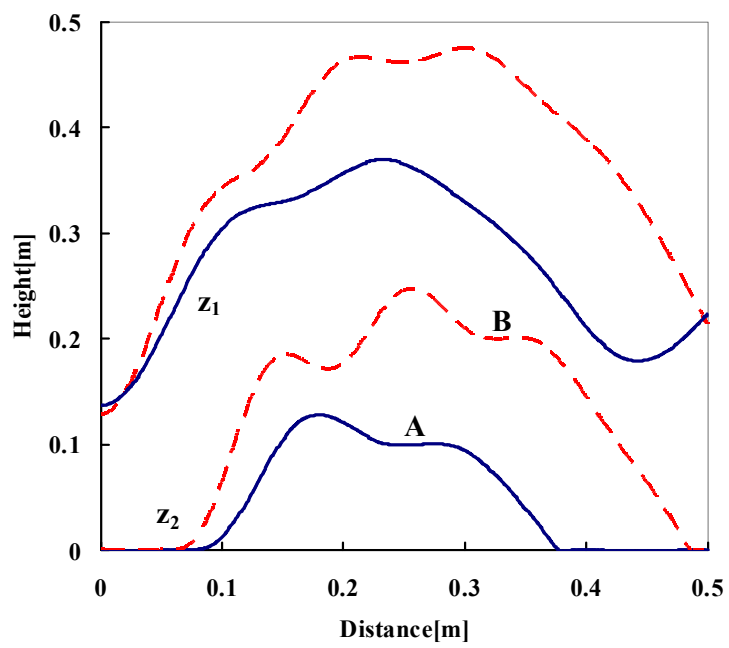

Fig. 3. Two typical examples of trajectories of mass points, $z_{1}$ and $z_{2}$, during hopping 


\section{Hopping properties}

In this section, we present the characteristics during the hopping motion. The trajectories of the robot change, depending mainly on passive parameters such as reduced mass, the mass ratio between the upper and lower masses, and the spring constant and active parameters such as initial spring contraction, wire tension, and horizontal traveling driving force. Passive parameters are mechanical design parameters and should be designed a priori for the specification of the stairs in an office building. On the other hand, active parameters are control parameters and can be changed in accordance with a local irregular step, etc.

We first show the frequency characteristic, one of the characteristics of the passive parameters, during the hopping motion. Figure 4 shows the relation between the reduced mass and the angular frequency for three spring constants. Here, the lines depict the results obtained by Eq. (3c) and the points are the results performed by 10 hopping experiments for each point. The passive parameters are as follows: The spring constants, $k$, are 800, 1,200, and $1,600 \mathrm{~N} / \mathrm{m}$. In the experiments, reduced masses, $M$, of $0.4,0.5,0.6,0.7$ and $0.8 \mathrm{~kg}$ are used. Here, when the reduced mass is $0.6 \mathrm{~kg}$ and the mass ratio is 2.0 , the masses of the upper and lower body parts mean $1.8 \mathrm{~kg}$ and $0.9 \mathrm{~kg}$, respectively, and the total mass is 2.7 $\mathrm{kg}$. This figure implies that the angular frequency can almost be controlled by $\omega$ in Eq. (3c), that is, the reduced mass and the spring constant, because, although the experimental values became slightly higher than the simulation results in accordance with the increase in the spring constant, the errors can be estimated simply from the figure and the standard deviations were also very small.

Second, we show the trajectories of body parts 1 and 2 for mass ratios, $m_{1} / m_{2}$, of 0.5 (Case A) and 4.0 (Case B), as two typical examples obtained in numerical simulations (Fig. 5). Here, the reduced mass, $M$, is constant at $0.7 \mathrm{~kg}$, the spring constant, $k$, is $2,000 \mathrm{~N} / \mathrm{m}$, and the initial contraction of the spring, $h$, is $0.1 \mathrm{~m}$. The thin lines represent body part 1 and the bold lines are body part 2 . The dashed thin lines represent the velocities of body part 1 and the dashed bold lines are the velocity of boby part 2 . This figure shows that the amplitudes of body parts 1 and 2 depend on the mass ratio, as shown by the vibration term in Eq. (3b). That is, if the reduced mass is constant, the amplitude of body part 1 is large when the mass of body part 2 is large, and the amplitude of body part 2 is large when the mass of body part 1 is large. In addition, the hopping height of the COM increases with decreasing the mass of body part $2, m_{2}$. Furthermore, the possible points of soft-landing $\left(\mathrm{z}_{2}^{\prime} \approx 0, \mathrm{z}_{2}^{\prime \prime} \approx 0\right.$, and $\left.\mathrm{z}_{2}^{\prime \prime \prime} \leq 0\right)$ exist in the neighborhood of crests of the vibration of body part 2 , as shown in Cases $A$ and B. Note that we cannot choose the highest hopping point, i.e., a vertex, as the landing point, because the robot has a physical body length, $L$, and cannot land both the front and rear wheels on the stairs simultaneously. Additionally, we also cannot reduce the mass of body part 2 dramatically, as a main drive unit such as a motor is mounted on body part 2 .

Next, as active hopping characteristics, Fig. 6 shows examples of the hopping motion for the initial contractions of the spring of $h=0.070,0.085$, and $0.100[\mathrm{~m}]$. Here, the reduced mass, $M$, is $0.7 \mathrm{~kg}$, the mass ratio, $m_{1} / m_{2}$, is 2.0 , the spring constant, $k$, is $1,600 \mathrm{~N} / \mathrm{m}$ and the horizontal velocity, $v_{\mathrm{x}}$, is $1.2 \mathrm{~m} / \mathrm{s}$. From this, we find that with the increase in the initial contraction of the spring, $h$, the temporal axis of the parabolic motion of the COM, T, is shifted to the right, the hopping height of the COM is increased, but the angular frequency, $\omega$, is unchanged. Also, we can simply find that the spatio-frequency represented by $x-z$ coordinates can be controlled by the horizontal velocity $v_{x}$. The slower the horizontal velocity, the higher the spatio-frequency. The correspondence between the spatio-frequencies of the lower body 
part trajectory and the stair configuration, i.e., the tread-riser interval, enables economic and stable stair climbing by the robot. Moreover, as the wire can pull the body parts mutually, the robot can actively control the possible point of soft landing by the wire tension.

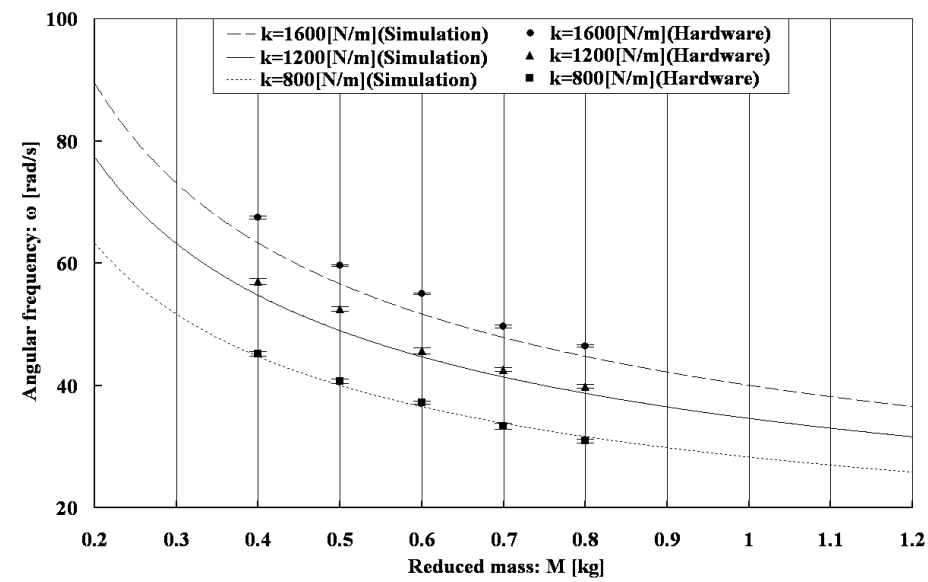

Fig. 4. Relation between reduced mass and angular frequency for different spring constants
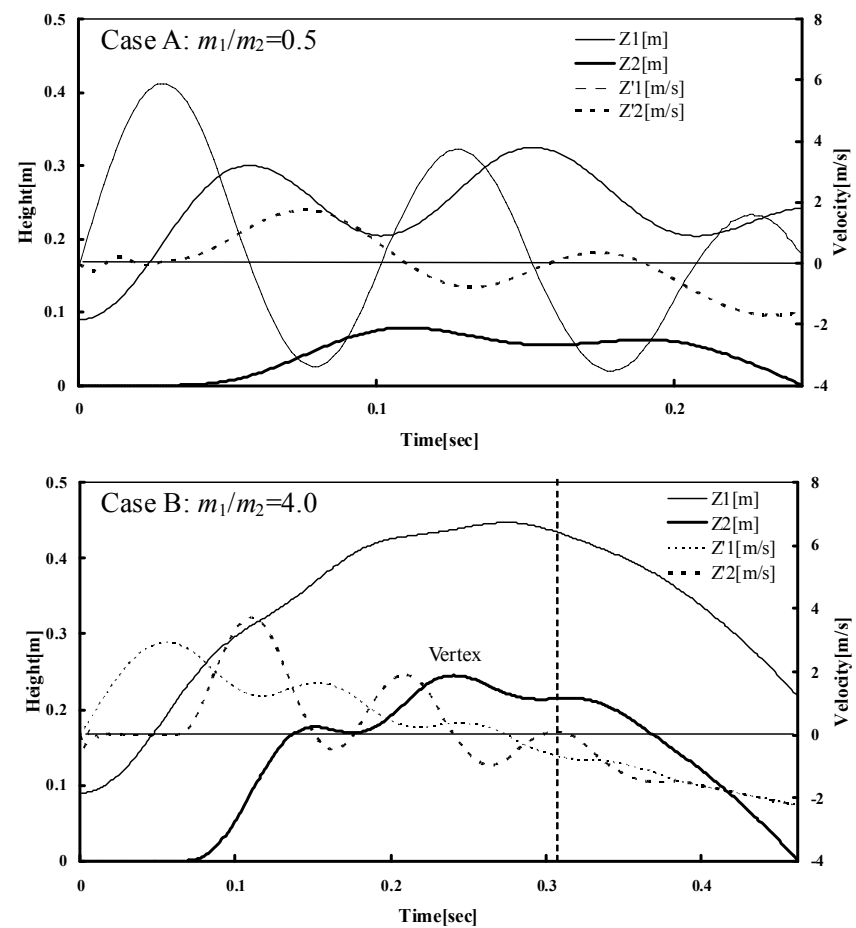

Fig. 5. Trajectories of mass and velocities: in Case A, the mass ratio is 0.5 , and in Case B, the mass ratio is 4.0 


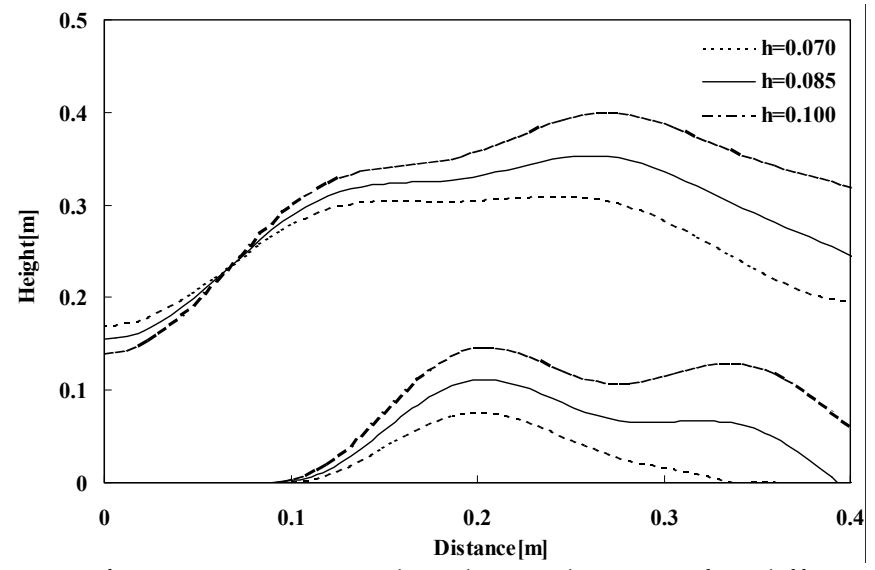

Fig. 6. Trajectories of mass points 1 and 2 during hopping for different initial spring contractions

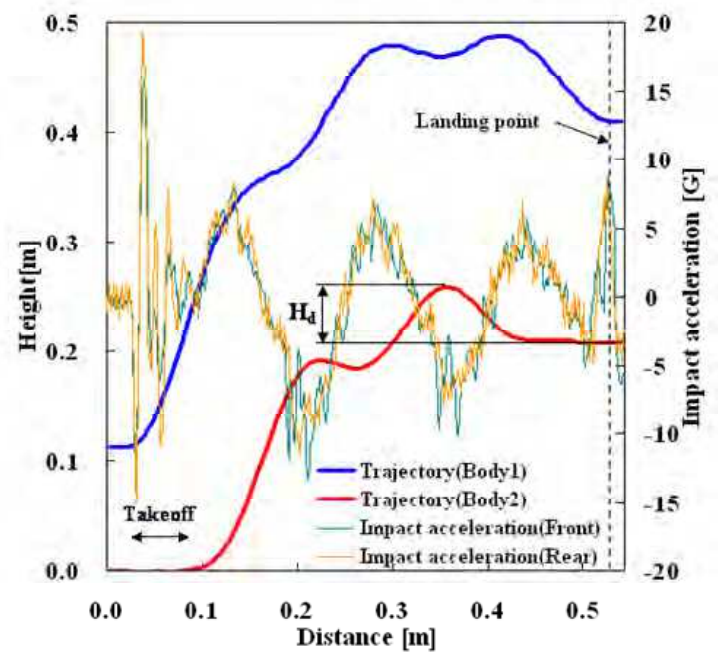

Fig. 7. Trajectories of two body parts and impact accelerations during hopping motion

\section{Demonstration of stair climbing}

\subsection{Climbing up a step}

We first demonstrate fast and soft climbing up a riser $0.21 \mathrm{~m}$ in height. From the above discussion, we can control the landing point and condition by adjusting the mechanical design and control parameters. Here, we set the parameters as follows: the reduced mass, $M$, of $0.55 \mathrm{~kg}$, the mass ratio, $m_{1} / m_{2}$, of 1.32 , the spring constant, $k$, of $1,600 \mathrm{~N} / \mathrm{m}$, the initial contraction of the spring, $h$, of $0.13 \mathrm{~m}$, and the horizontal velocity, $v_{x}$, of $1.2 \mathrm{~m} / \mathrm{s}$. Note that the value of $0.21 \mathrm{~m}$ is 7.5 times higher than the wheel radius of the robot and corresponds approximately to the common riser height of stairs. Also, the contraction of the spring, $h$, is 
measured by the PSD with a sampling time of $30 \mathrm{~ms}$. Figure 7 shows the trajectories of the robot during the hopping motion and the impact accelerations obtained from the front and rear sensors mounted in the lower body part. The impact acceleration at the moment of landing was approximately $8 \mathrm{G}$, which was less than the maximum acceleration during takeoff, $19 \mathrm{G}$, and was close to that experienced during flight, i.e., almost $10 \mathrm{G}$. As the impact acceleration by free-fall from the maximum hopping height to the step $\left(H_{\mathrm{d}}\right.$ shown in Fig. 7) was approximately $33 \mathrm{G}$, the soft-landing of this robot reduced the impact by $76 \%$. However, high impact acceleration at the moment of takeoff was observed, unfortunately. This is not due to impact with the ground, but rather due to the plate deflection of body part 2. The actual acceleration of lower body part at the moment of takeoff was less than $10 \mathrm{G}$. We need to improve the geometrical moment of inertia of body part 2. Note that we realized fast and soft step climbing by $0.27 \mathrm{~m}$ in height.

\subsection{Climbing up a flight of stairs}

Next, we demonstrate fast and soft stair climbing. The trick in stair climbing is to synchronize the spatio-frequency of the stairs, i.e., the tread-riser intervals, and the body vibration. It is simple and easy in a mathematical model, but it is not in practice. Figure 8 shows three processes for continuous hopping-takeoff, landing, and reeling in-and the tread length required for each hop. The horizontal traveling distance during these three processes, the required tread length, can be quite simply controlled by the horizontal velocity $v_{x}$, if the robot length, $L$, is zero. However, the following constraints exist in practice. First, after takeoff, the front wheels must jump up to the edge of the step (takeoff phase in Fig. 8), next, before landing, the rear wheels must clear the edge of the step (landing phase in Fig. 8), and then the robot must reel in the wire for next hopping (reeling-in phase in Fig. 8). When the horizontal traveling distance during these three phases, $D_{T}+D_{L}+D_{R}$, is equal to or less than the tread length, the robot can climb a flight of stairs. Thus, this is the minimum required tread length, and the shorter, the better. From these constraints, the robot must jump up to the riser height, $H$, at $t=D_{\mathrm{T}} / v_{\mathrm{x}}$ and the minimum landing phase distance, $D_{\mathrm{L}}$, is equal to the body length, $L$. Also, since the reeling-in phase distance, $D_{R}$, depends only on the motor torque to reel in the wire, the larger the motor torque, the shorter the reeling-in phase distance. However, as the exceedingly high-power motor makes the upper mass heavy and the wire tension strong, it lifts up the lower body part. Additionally, although the reeling-in phase distance can be shortened by reeling in the wire before landing, the control of the soft landing point becomes difficult as the passive vibration characteristics change. Thus, the wire is reeled in after landing for simplification in this experiment.

Figure 9 shows an example of trajectories of two body parts (blue and red lines) and impact accelerations (green and orange lines) based on the following parameters: the reduced mass, $M$, of $0.79 \mathrm{~kg}$, the mass ratio, $m_{1} / m_{2}$, of 2.17 , the spring constant, $k$, of $2,000 \mathrm{~N} / \mathrm{m}$, the initial spring constriction, $h$, of $0.12 \mathrm{~m}$, and the horizontal velocity, $v_{x}$, of $0.90 \mathrm{~m} / \mathrm{s}$ for the riser height, $H$, of $0.20 \mathrm{~m}$. Additionally, Fig. 10 shows stroboscopic pictures of continuous hopping to climb two steps. As shown in Fig. 9, the required tread length was $0.74 \mathrm{~m}$ (Note that to avoid clashing into the riser wall, a margin safety of 2.1 was introduced. The required tread length obtained by the numerical simulation was $0.35 \mathrm{~m}$ ). The impact accelerations were $28 \mathrm{G}$ and $37 \mathrm{G}$ for first and second takeoffs and $10 \mathrm{G}$ and $6 \mathrm{G}$ for first and second landings. The impact acceleration at the moment of landing was less than that during flight. 
Also, the stair climbing time per step was $0.77 \mathrm{~s}$. To shorten the required tread length is one of the future tasks as the common tread length is almost $0.4 \mathrm{~m}$.

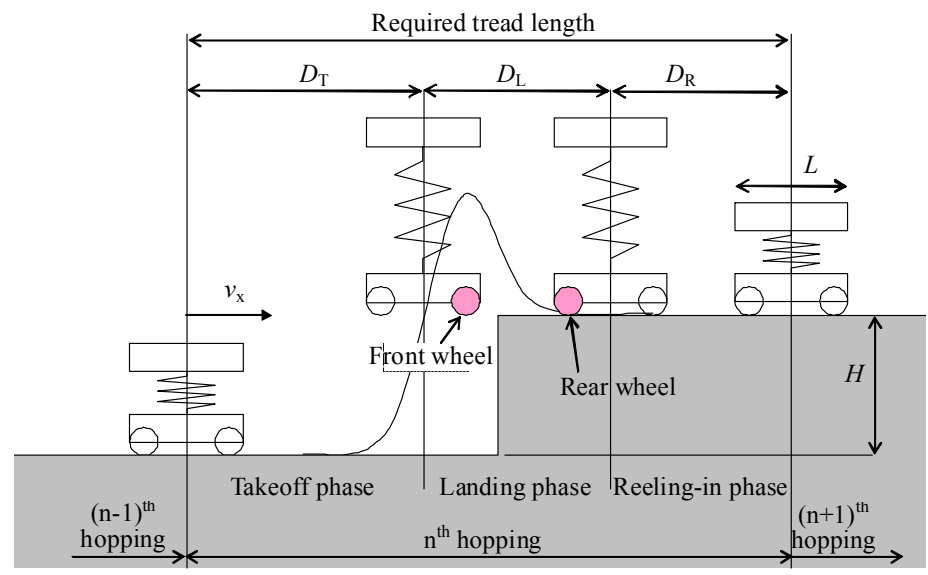

Fig. 8. Hopping processes and required tread length

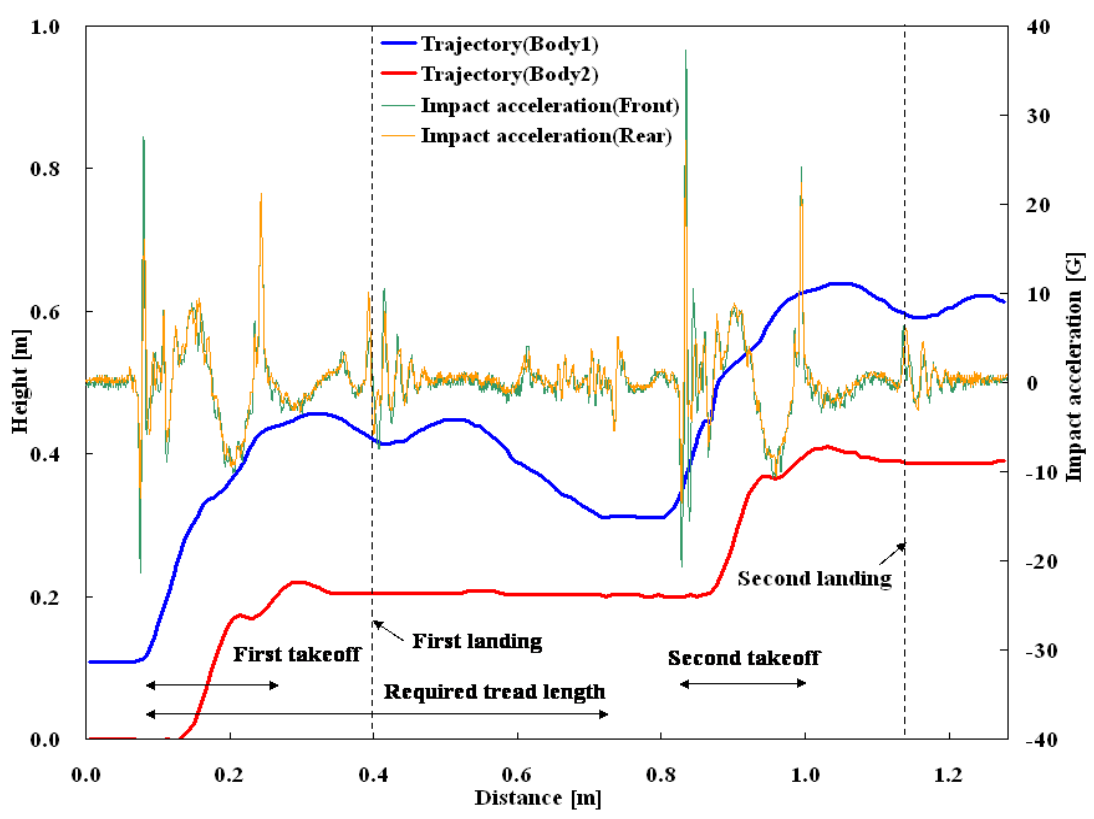

Fig. 9. Trajectories of two body parts and impact acceleration during stair climbing 

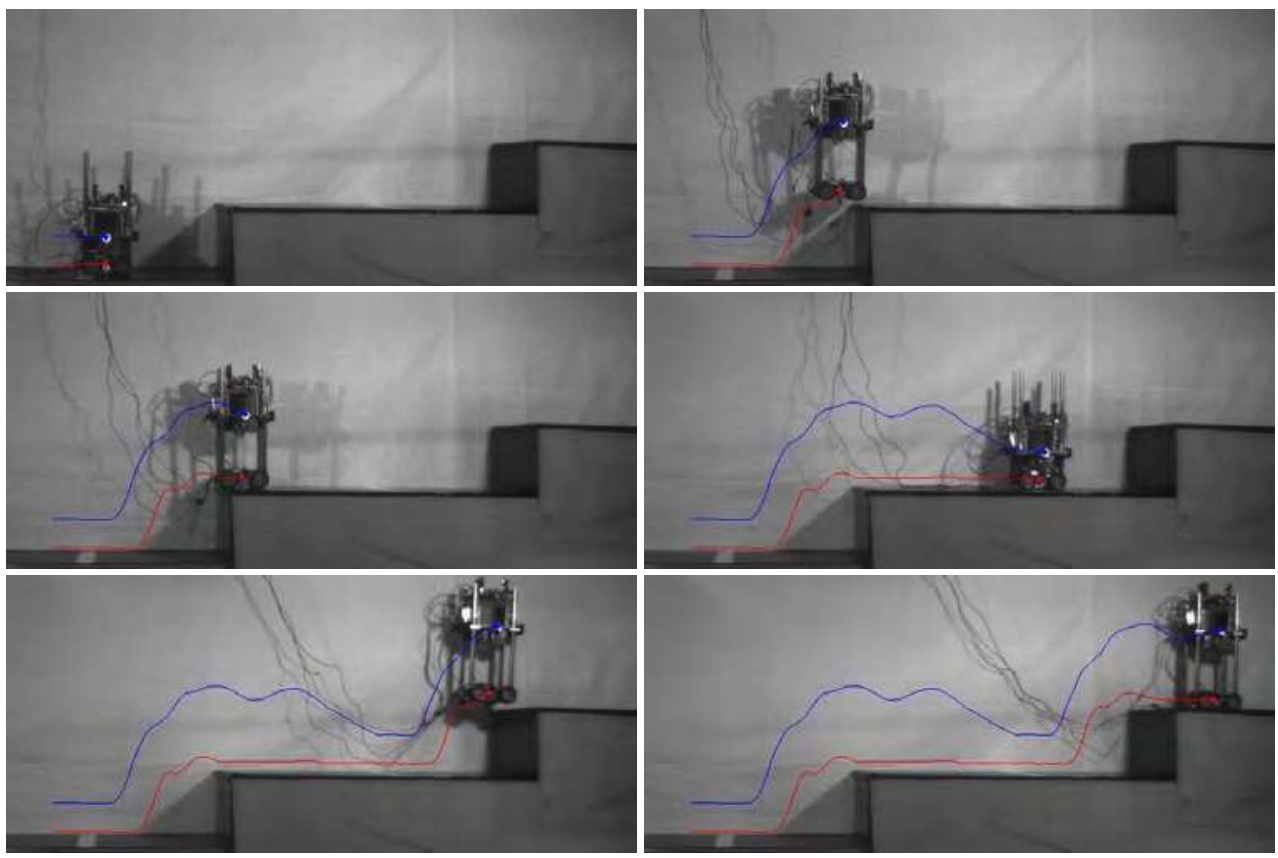

Fig. 10. Stroboscopic images of stair climbing

\section{Demonstration of step descending}

Finally, we demonstrate fast and soft descending of steps $0.20 \mathrm{~m}$ in height. The hopping mechanism is almost the same as for climbing. However, the degree of difficulty is quite different. As mentioned in Section 3, the soft-landing point is the location at which the velocity in the z-direction of vibration of lower body part, $-h M \omega / m_{2} \cos (\omega t+\varphi)$, and that of the parabolic motion of the COM, $-g(t-T)$, are canceled out. Here, although the maximum of the former is $h \mathrm{M \omega} / \mathrm{m}_{2}$, the latter becomes a large negative value with time, $t$, because of descending. In climbing, as the robot lands near the top of the parabolic motion, as shown in Fig. 8, and the descending velocity by parabolic motion is low, there are many parameters, $h M \omega / m_{2} \cos (\omega t+\varphi)$, which can cancel out the descending velocity. In contrast, in descending, as the robot lands considerably below the top of the parabolic motion, as the dashed line shows in Fig. 11, and the descending velocity is very high, the parameters, $h M \omega / m_{2}$ $\cos (\omega t+\varphi)$, which can cancel it out, decrease dramatically. Thus, we use another technique in descending. Hence, the robot does not jump up, but glides from the step horizontally, starts to vibrate by detaching the reel mechanism while descending, and then lands softly, as the solid line shows in Fig. 11. This method requires posture control at takeoff, but decreases the descending velocity by the parabolic motion on landing and makes the required tread length short.

Figure 12 shows the trajectories of two body parts (blue and red lines) and impact accelerations (green and orange lines) during the hopping motion. Here, the parameters are: the reduced mass, $M$, of $0.74 \mathrm{~kg}$, the mass ratio, $m_{1} / m_{2}$, of 2.04 , the spring constant, $k$, of 
$1,200 \mathrm{~N} / \mathrm{m}$, the initial contraction of the spring, $h$, of $0.11 \mathrm{~m}$, and the horizontal velocity, $v_{x}$, of $1.0 \mathrm{~m} / \mathrm{s}$. The impact acceleration at the moment of landing was approximately $14 \mathrm{G}$, which was close to that experienced during flight, i.e., almost $10 \mathrm{G}$. As the impact acceleration under free-fall from the riser height to the step was $77 \mathrm{G}$, the soft-landing of this robot reduced the impact by $82 \%$. Figure 13 shows stroboscopic images of step descending. The posture at takeoff was controlled by a wheelie.

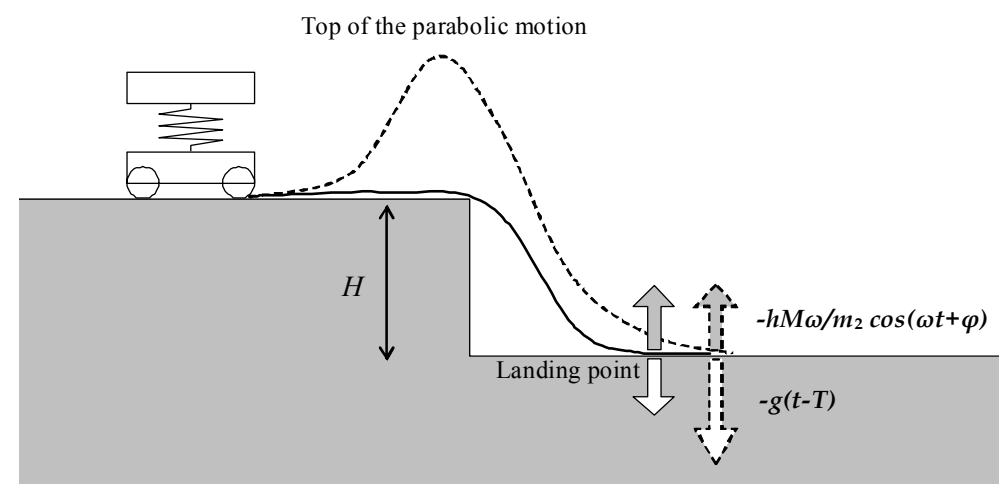

Fig. 11. Two methods for descending stairs

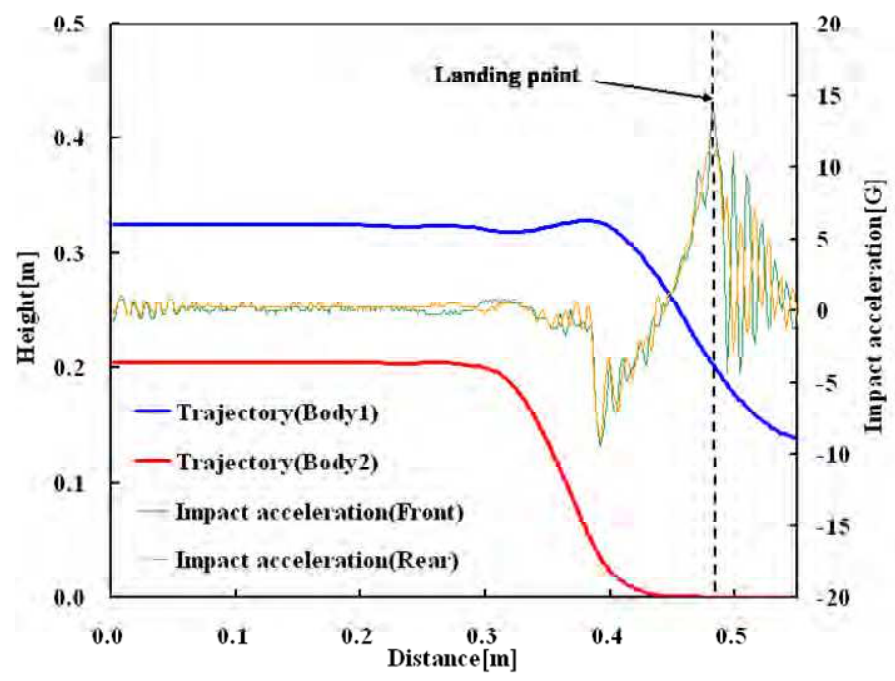

Fig. 12. Trajectories of the two body parts and impact accelerations during hopping motion 

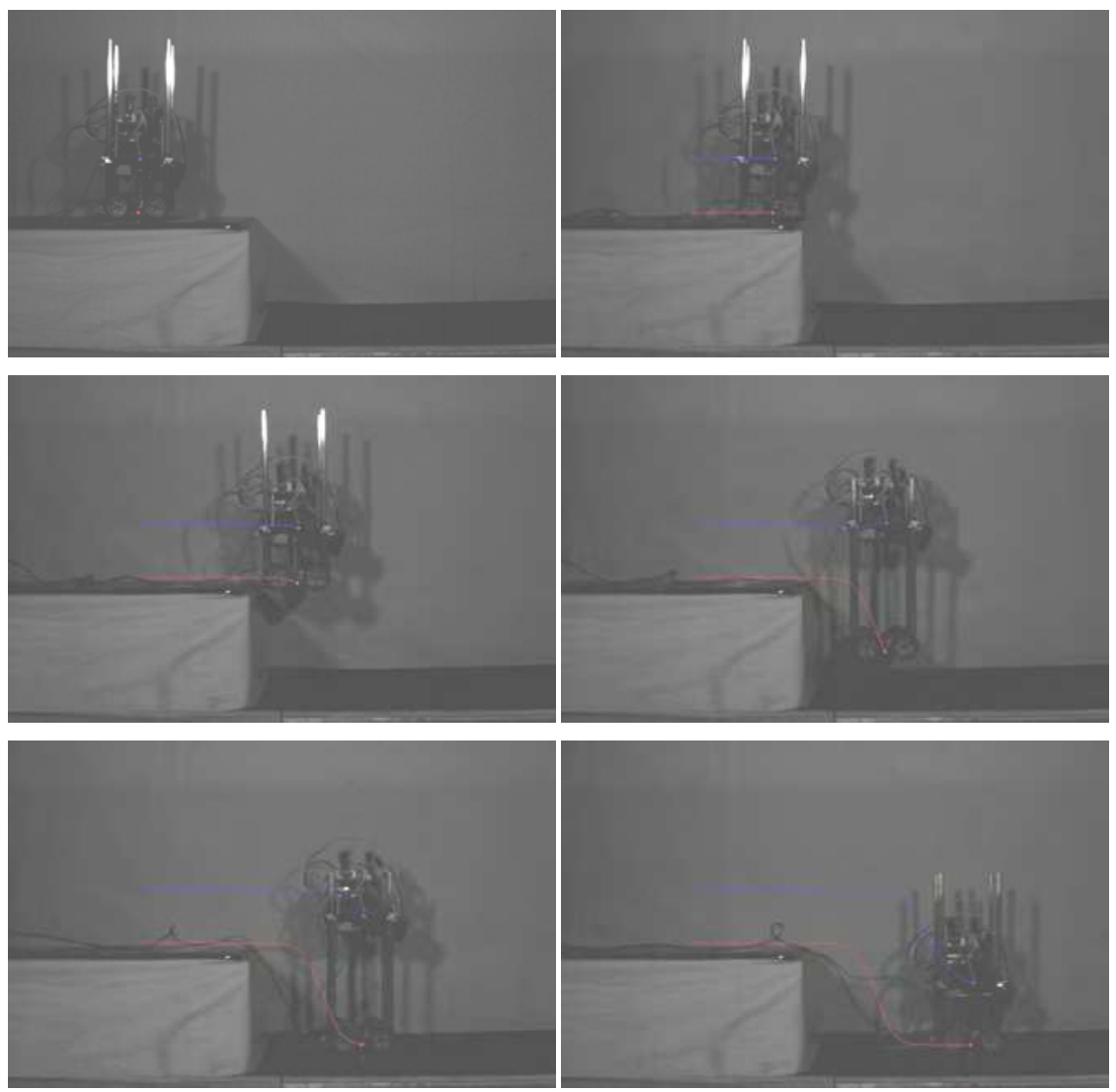

Fig. 13. Stroboscopic images of step descending

\section{Conclusion}

We introduced a wheel-based stair-climbing robot with a hopping mechanism for stairclimbing. The robot, consisting of two body parts connected by springs, climbed stairs quickly, softly, and economically by using the vibration of a two-degrees-of-freedom system. In the future, we intend to shorten the required tread length by controlling the wire tension and minimizing the body length to realize a practical stair-climbing robot. 


\section{References}

Altendorfer, R.; Moore, E.Z.; Komsuoglu, H.; Buehler, M.; Brown, H.; McMordie, D.; Saranli, U.; Full, R. \& Koditschek, D.E. (2001). A Biologically Inspired Hexapod Runner, Autonomous Robots, Vol. 11, (month 2001), pp. 207 - 213

Asai, Y.; Chiba, Y.; Sakaguchi, K.; Sudo, T.; Bushida, N.; Otsuka, H.; Saito, Y. \& Kikuchi, K. (2008). Wheel-Based Stair-climbing Robot with Hopping Mechanism Demonstration of Continuous Stair Climbing Using Vibration-, Journal of Robotics and Mechatronics, Vol. 20, No. 2, Apr. 2008, pp. 221-227

ASIMO OFFICIAL SITE : http://www.honda.co.jp/ASIMO/

Harada, K.; Kajita, S.; Kaneko, K. \& Hirukawa, H. (2006). Dynamics and Balance of a Humanoid Robot during Manipulation Tasks, IEEE Transaction on Robotics, 2006, vol. 22, no. 3, pp. 568-575.

Hirose, S.; Sensu, T. \& Aoki, S. (1992). The TAQT Carrier: A Practical Terrain-Adaptive Quadru-Track Carrier Robot, Proceedings of IEEE/RSJ International conference on Intelligent Robots and Systems, July 1992, pp. 2068-2073, Tokyo

Kikuchi, K.; Sakaguchi, K.; Sudo, T.; Bushida, N.; Chiba, Y. \& Asai, Y. (2008). A study on wheel-based stair-climbing robot with hopping mechanism, MECHANICAL SYSTEMS AND SIGNAL PROCESSING (MSSP), Aug. 2008, Vol. 22, Issue 6, $1316-$ 1326, ELSEVIER

Matsumoto, O.; Kajita, S.; Saigo, M. \& Tani, K; (1999). Biped-type leg-wheeled robot, Advanced Robotics, 13(3), Oct. 1999, pp.235-236.

Nakajima, S.; Nakano, E.; \& Takahashi, T.; (2007). Motion Control Technique for Practical Use of a Leg-Wheel Robot on Unknown Outdoor Rough Terrains, Proceedings of IEEE/RSJ International conference on Intelligent Robots and Systems, vol.1, (Month 2004), pp. 1353-1358

Sakaguchi, K.; Sudo, S.; Bushida, N.; Chiba, Y.; Asai, Y. \& Kikuchi, K. (2007). Wheel-Based Stair-climbing Robot with Hopping Mechanism -Fast Stair-climbing and Softlanding by Vibration of 2-DOF system-, Journal of Robotics and Mechatronics, Vol. 19, No. 3, Jun. 2007, pp. 258-263

Sugahara, Y.; Carbone, G.; Hashimoto, K.; Ceccarelli, M.; Lim, H. \& Takanishi, A. (2007). Experimental Stiffness Measurement of WL-16RII Biped Walking Vehicle during Walking Operation, Journal of Robotics and Mechatronics, Vol. 19, No. 3, Jun. 2007, pp. 272-280

Yim, M. H.; Homans, S. B. \& Roufas, K. D. (2001). Climbing with snake-like robots, IFAC Workshop on Mobile Robot Technology, Korea, May 2001, pp. 21-22, Jejudo.

Yoshida, T.; Koyanagi, E.; Tadokoro, E.; Yoshida, K.; Nagatani, K.; Ohno, K.; Tsubouchi, T.; Maeyama, S.; Noda, I.; Takizawa, O. \& Hada, Y. (2007). A High Mobility 6-Crawler Mobile Robot "Kenaf", Proceedings of 4th International Workshop on Synthetic Simulation and Robotics to Mitigate Earthquake Disaster (SRMED2007), July, 2007, p. 38, Atlanta 


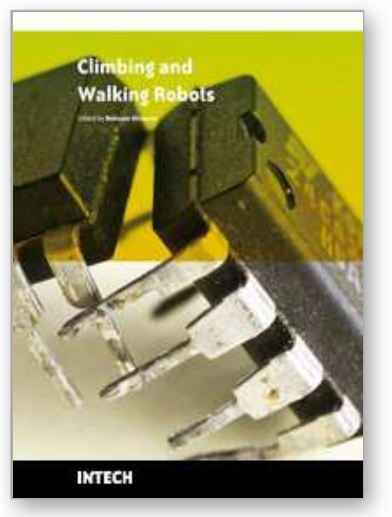

\author{
Climbing and Walking Robots \\ Edited by Behnam Miripour
}

ISBN 978-953-307-030-8

Hard cover, 508 pages

Publisher InTech

Published online 01, March, 2010

Published in print edition March, 2010

Nowadays robotics is one of the most dynamic fields of scientific researches. The shift of robotics researches from manufacturing to services applications is clear. During the last decades interest in studying climbing and walking robots has been increased. This increasing interest has been in many areas that most important ones of them are: mechanics, electronics, medical engineering, cybernetics, controls, and computers. Today's climbing and walking robots are a combination of manipulative, perceptive, communicative, and cognitive abilities and they are capable of performing many tasks in industrial and non- industrial environments. Surveillance, planetary exploration, emergence rescue operations, reconnaissance, petrochemical applications, construction, entertainment, personal services, intervention in severe environments, transportation, medical and etc are some applications from a very diverse application fields of climbing and walking robots. By great progress in this area of robotics it is anticipated that next generation climbing and walking robots will enhance lives and will change the way the human works, thinks and makes decisions. This book presents the state of the art achievments, recent developments, applications and future challenges of climbing and walking robots. These are presented in 24 chapters by authors throughtot the world The book serves as a reference especially for the researchers who are interested in mobile robots. It also is useful for industrial engineers and graduate students in advanced study.

\title{
How to reference
}

In order to correctly reference this scholarly work, feel free to copy and paste the following:

Koki Kikuchi, Naoki Bushida, Keisuke Sakaguchi, Yasuhiro Chiba, Hiroshi Otsuka, Yusuke Saito, Masamitsu Hirano and Shunya Kobayashi (2010). A Wheel-based Stair-climbing Robot with a Hopping Mechanism, Climbing and Walking Robots, Behnam Miripour (Ed.), ISBN: 978-953-307-030-8, InTech, Available from: http://www.intechopen.com/books/climbing-and-walking-robots/a-wheel-based-stair-climbing-robot-with-ahopping-mechanism

\section{INTECH}

open science | open minds

\section{InTech Europe}

University Campus STeP Ri

Slavka Krautzeka 83/A

51000 Rijeka, Croatia

Phone: +385 (51) 770447

\section{InTech China}

Unit 405, Office Block, Hotel Equatorial Shanghai

No.65, Yan An Road (West), Shanghai, 200040, China

中国上海市延安西路65号上海国际贵都大饭店办公楼 405 单元

Phone: +86-21-62489820 
Fax: +385 (51) 686166

Fax: +86-21-62489821

www.intechopen.com 
(C) 2010 The Author(s). Licensee IntechOpen. This chapter is distributed under the terms of the Creative Commons Attribution-NonCommercialShareAlike-3.0 License, which permits use, distribution and reproduction for non-commercial purposes, provided the original is properly cited and derivative works building on this content are distributed under the same license. 